\title{
Evaluation of Spectrum Occupancy in Spain for Cognitive Radio Applications
}

\author{
Miguel López-Benítez, Anna Umbert and Fernando Casadevall \\ Department of Signal Theory and Communications \\ Universitat Politècnica de Catalunya (UPC) \\ Barcelona, Spain \\ [miguel.lopez, annau, ferranc]@tsc.upc.edu
}

\begin{abstract}
Cognitive Radio (CR) has been identified as a promising solution to the so-called spectrum scarcity problem. The basic idea of this paradigm is to allow unlicensed users to access in an opportunistic and non-interfering manner some licensed bands that are temporarily unoccupied by licensed users. CR is expected to significantly increase current spectrum usage. However, before this paradigm can turn into reality, a full understanding of the dynamic usage of spectrum is required. Current spectrum utilization has already been evaluated in some measurement campaigns. However, most of them were performed in USA and therefore evaluated the American spectrum regulation and utilization. Few studies have been carried out in other places. In this context, this paper presents spectrum occupancy measurements conducted in the frequency range from $75 \mathrm{MHz}$ to $3 \mathrm{GHz}$ in an outdoor environment in urban Barcelona, Spain. The measurements are analyzed and compared to the official spectrum regulations. The obtained results demonstrate the existence of a significant amount of spectrum available for the deployment of future CR networks.
\end{abstract}

Keywords - cognitive radio; dynamic spectrum access; measurement campaign; spectrum occupancy; wireless networks.

\section{INTRODUCTION}

Cognitive Radio (CR) has been paid much attention in both academic and industrial spheres during the last years for its capability to solve the conflicts between spectrum demand growth and spectrum underutilization [1]. The basic principle of the CR paradigm is to allow unlicensed opportunistic access to temporarily unused licensed frequency bands provided that no harmful interference is caused to legitimate licensed users. This paradigm will result in increased spectrum usage efficiency. Nevertheless, before investigating the technical issues of this type of communication systems, it becomes necessary to have a clear picture of how different frequency bands are used in specific environments. An appropriate study of the current spectrum usage will enable the characterization of temporarily unoccupied spectral resources in terms of frequency, time, and space, as well as the identification of the most suitable and interesting bands for Cognitive Radio Networks (CRNs). Therefore, the measurement of real network activities constitutes an important step towards a realistic understanding of dynamic spectrum utilization and hence towards the deployment of future CRNs.
Several measurement campaigns covering both wide frequency ranges [2]-[4] as well as some specific licensed bands [5]-[9] have already been performed in diverse locations and scenarios in order to determine the degree to which allocated spectrum bands are occupied in real wireless communication systems. However, most of them were carried out in USA and therefore analyzed the American spectrum regulation and utilization. Few studies have been carried out in other places. Some examples are the measurements performed in New Zealand [10], Singapore [11] and in some European cities such as Dublin, Ireland [3] and Aachen, Germany [12]. All these spectrum measurements provide a valuable source of information for a realistic understanding of dynamic spectrum utilization and practical development of the CR technology. However, in order to enable a wide scale deployment, the CR technology cannot be developed based on the conclusions derived from the studies conducted in a few geographical areas or under a specific spectrum regulation. CR should take into account the possibility to operate under many different spectrum regulations and a wide variety of scenarios. Further spectrum measurements are therefore required.

In this context, the Radio Communications Group of the Technical University of Catalonia (Universitat Politècnica de Catalunya) is carrying out a broadband spectrum measurement campaign that will cover the frequency range from $75 \mathrm{MHz}$ to $7 \mathrm{GHz}$ under both indoor and outdoor scenarios in urban and suburban locations in Barcelona, Spain. This paper presents the results obtained in an initial phase of the campaign that covers the frequency range between $75 \mathrm{MHz}$ and $3 \mathrm{GHz}$ in an outdoor urban environment. The measurements are analyzed in order to determine the spectrum occupancy and compared to the official spectrum regulations defined by the governmental agency in charge of the frequency allocations in Spain [13]. The obtained results demonstrate the existence of a significant amount of spectrum available for the deployment of future CRNs.

The remainder of this paper is organized as follows. First, section II describes the equipment employed to perform the measurements reported in this paper, its configuration and the measurement methodology that has been followed. The obtained measurement results are presented and analyzed in section III. Finally, section IV highlights the main conclusions derived from this study and describes the planned future work. 


\section{Measurement Setup And Methodology}

The equipment employed in this study consisted of a broadband discone antenna AOR DN753 (vertically polarized with omni-directional receiving pattern in the horizontal plane) specified for the frequency range $75 \mathrm{MHz}-3 \mathrm{GHz}$, which was connected with a 10-meter low loss coaxial cable RG-58A/U to a high performance handheld spectrum analyzer Anritsu Spectrum Master MS2721B. The spectrum analyzer was controlled by a laptop connected via a cross-over Ethernet cable. The measurement setup is shown in Figure 1.

During the measurements the antenna was placed on the roof of the Department's building in Campus Nord (latitude: 41 23 ' 20" north; longitude: $2^{\circ}$ 6' 43” east; altitude: 175 meters). This is a strategic location with direct line-of-sight to several transmitting stations located a few tens or hundreds of meters away from the antenna and without buildings blocking the radio propagation. This strategic location enabled us to accurately measure the spectral activity of, among others, a TV repeater, a FM broadcast station, several nearby base stations for cellular mobile communications and a military headquarter as well as some potential maritime transmitters due to the relative proximity to the Barcelona's harbor.

The main configuration parameters used for the spectrum analyzer are shown in TABLE I. The measured frequency range was divided into six consecutive $500 \mathrm{MHz}$ blocks. Each block was measured during a continuous period of 48 hours (more than 10000 traces were obtained for each block). The measured traces were saved in an external storage device and post-processed off-line using MATLAB in a powerful PC.

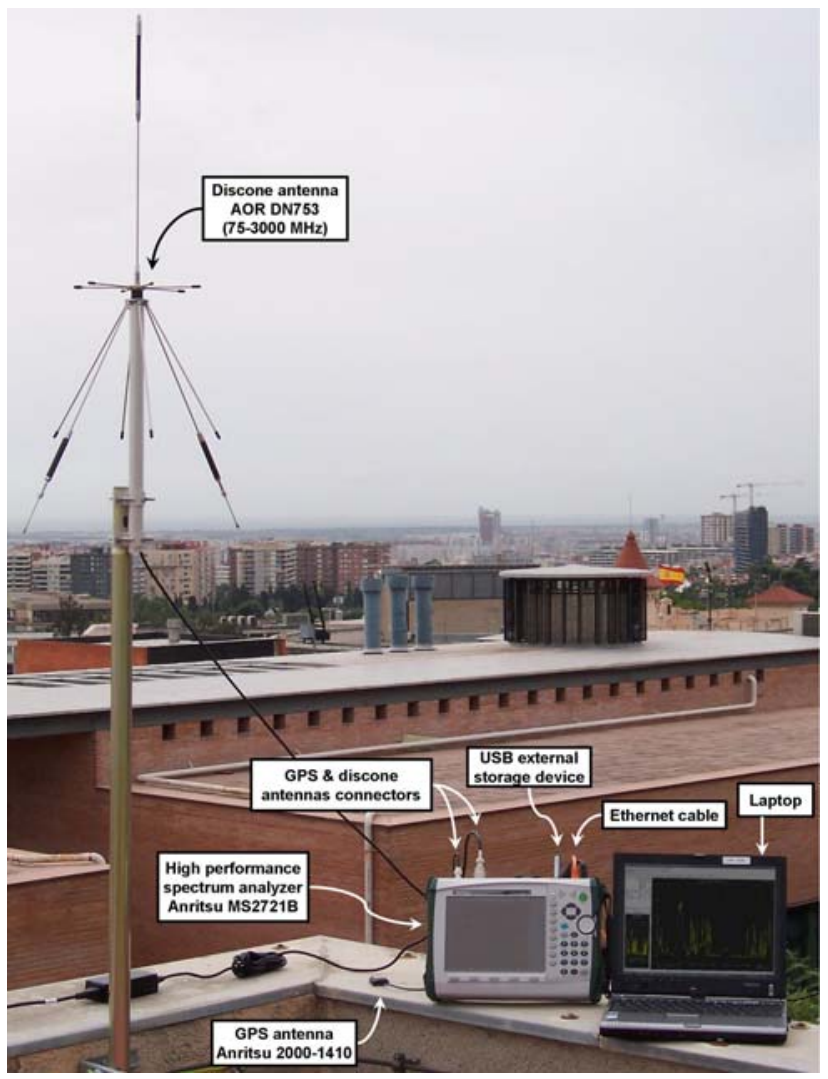

Figure 1. Measurement equipment employed in this study.
TABLE I. SPECTRUM ANALYZER CONFIGURATION.

\begin{tabular}{|l|l|}
\hline \multicolumn{1}{|c|}{ Parameter } & \multicolumn{1}{c|}{ Value } \\
\hline Center frequency & $\begin{array}{l}\text { Block 1: } 250 \mathrm{MHz} \quad \text { Block 4: } 1750 \mathrm{MHz} \\
\text { Block 2: } 750 \mathrm{MHz} \quad \text { Block 5: 2250 MHz } \\
\text { Block 3: } 1250 \mathrm{MHz} \quad \text { Block 6: } 2750 \mathrm{MHz}\end{array}$ \\
\hline Frequency span & $500 \mathrm{MHz}$ \\
\hline $\begin{array}{l}\text { Resolution/video } \\
\text { bandwidth (RBW/VBW) }\end{array}$ & $10 \mathrm{kHz} / 10 \mathrm{kHz}$ \\
\hline Sweep time & Automatically selected \\
\hline Reference level & $-20 \mathrm{dBm}$ \\
\hline Scale & $10 \mathrm{~dB} /$ div \\
\hline Detection type & RMS detector \\
\hline
\end{tabular}

\section{MEASUREMENT RESUlts AND ANALYSIS}

\section{A. Occupancy Metrics}

The obtained measurement results are shown in Figures 2 and 3. Each figure is composed of three graphs, each one corresponding to a specific occupancy metric. The upper graph shows the minimum, maximum and average Power Spectral Density (PSD) measured for each $500 \mathrm{MHz}$ spectrum block. The middle graph shows the temporal evolution of the spectrum occupancy during the whole 48 hour measurement period. A black dot indicates that the corresponding frequency point was measured as occupied at that time instant, while the white color means that the frequency point was measured as idle. To determine whether a given frequency is occupied or not, energy detection is assumed. Different sensing methods have been proposed in the literature to detect whether a frequency band is being used by a licensed user [14]. They provide different trade-off between required sensing time, complexity and detection capabilities. Depending on how much information is available about the signal used by the licensed network different performances can be reached. However, in the most generic case no prior information is available about the licensed signal. In such a case, the energy detection method is the only possibility left. Energy detection compares the received signal energy in a certain frequency band to a predefined threshold. If the signal is above the threshold, the band is determined to be occupied by the licensed network. Otherwise the band is supposed to be idle and could be used by a CRN. Following this principle, the measured PSD is compared to a given threshold. Measured samples above the threshold are assumed to be occupied; the rest of frequencies are assumed to be idle. To compute the decision threshold, the antenna was replaced by a matched load of $50 \Omega$ in order to measure the system's noise. At each frequency point the decision threshold was fixed such that only $1 \%$ of the measured noise samples lied above the threshold, which implies a false alarm probability of about $1 \%$. It is worth noting that the decision threshold obtained with this method is not constant since the system noise floor slightly increases with the frequency. Finally, the lower graph in each figure shows the duty cycle or percentage of time that each frequency point is measured as occupied by a licensed signal. The average duty cycle over the whole $500 \mathrm{MHz}$ spectrum block is also shown. 


\section{B. Analysis of Spectrum Occupancy}

The occupancy results shown in Figures 2 and 3 indicate that spectrum experiences a relatively high use below $1 \mathrm{GHz}$ while remains mostly underutilized between 1 and $3 \mathrm{GHz}$, with some exceptions such as the DCS 1800 system (1710-1785 $\mathrm{MHz}$ and $1805-1880 \mathrm{MHz}$ ) and the UMTS system (1920-1980 $\mathrm{MHz}$ and $2110-2170 \mathrm{MHz}$ ). In fact, while the average duty cycle between 75 and $1000 \mathrm{MHz}$ is 58.65\%, the value for this parameter between 1000 and $3000 \mathrm{MHz}$ is only $5.89 \%$, as shown in TABLE II. The overall average duty cycle over the whole frequency range considered in this study is $22.57 \%$, which indicates the existence of a significant amount of unused spectrum that could potentially be exploited by future CRNs. Although these results indicate low spectrum utilization, they do not provide a detailed description of how spectrum is used in different bands allocated to different specific services. Therefore, in the following we discuss in detail the spectrum usage in some allocated bands of interest.

TABLE II. AVERAGE DUTY CYCLE STATISTICS.

\begin{tabular}{|c|c|c|c|c|}
\hline \multirow{2}{*}{ Block } & $\begin{array}{c}\text { Frequency } \\
\text { range (MHz) }\end{array}$ & \multicolumn{2}{|c|}{ Average duty cycle } \\
\cline { 1 - 3 } 1 & $75-500$ & $60.98 \%$ & \multirow{2}{*}{$58.65 \%$} & \\
\cline { 1 - 2 } 2 & $500-1000$ & $56.67 \%$ & & \multirow{2}{*}{$22.57 \%$} \\
\cline { 1 - 2 } 3 & $1000-1500$ & $2.07 \%$ & \\
\cline { 1 - 2 } 4 & $1500-2000$ & $12.08 \%$ & \\
\hline 5 & $2000-2500$ & $7.57 \%$ & & \\
\hline 6 & $2500-3000$ & $1.85 \%$ & & \\
\hline
\end{tabular}

Although spectrum occupancy below $1 \mathrm{GHz}$ is relatively high, some opportunities for CRNs can be found. One example is the frequency band $470-862 \mathrm{MHz}$, which is allocated to analogic and digital terrestrial TV. The frequency range 830$862 \mathrm{MHz}$ assigned to digital TV exhibits an intensive usage that precludes CRN applications in this band. Moreover, several channels in the band $470-830 \mathrm{MHz}$ are also occupied. Note that occupied TV channels show a duty cycle about $100 \%$, i.e. continuous broadcasting, which impedes temporary opportunistic usage of those channels. However, the band 470$830 \mathrm{MHz}$ shows several TV channels that are received with very weak signal levels. The average duty cycle in this band is $66.58 \%$, which indicates that one third of the band (approximately $130 \mathrm{MHz}$ ) is unoccupied due to the weak reception of the signals broadcasted from those TV stations. Therefore, although this band is considerably populated it provides some interesting opportunities for secondary usage.

The E-GSM 900 band for uplink (880-915 MHz) appears as another potential candidate for CRN applications with an average duty cycle equal to $4.03 \%$. However, in this case it is important to highlight that the low activity recorded in this band does not necessarily imply that it could be used by CRNs. The considerably higher activity observed in the E-GSM 900 downlink (925-960 MHz) and the fact that GSM is based on FDD suggest that the actual usage of the E-GSM 900 uplink band might be higher than the activity level recorded by the spectrum analyzer. The unbalanced occupancy patterns observed between uplink and downlink in Figure 2 can be explained as follows. The transmission power of GSM base stations is considerably higher than that of cellular phones. Therefore, the presence of GSM downlink signals can be more easily detected. Moreover, the antenna employed in our study was placed on the roof of the Department's building with direct line-of-sight to several nearby base stations, which enabled us to accurately measure the high spectral activity of the downlink (96.20\%). On the other hand, the low usage observed in the uplink (4.03\%) may be due to the usually low transmission power of cellular phones and the resulting weak uplink signal received at the antenna. The detection of such signals might be hindered by the fact that cellular phones usually operate at the ground level or low altitudes and usually have no direct line-ofsight neither with the serving base station nor the antenna employed in our study, which makes more difficult to detect the activity in the uplink (the maximum PSD observed in the uplink band in Figure 2 may be due to phone calls from nearby locations, e.g. the upper floors of the Department's building). Therefore, from the obtained results we cannot conclude that there is a low activity level in the E-GSM 900 uplink band.

The frequency bands 235-273 MHz and 322-390 $\mathrm{MHz}$ show some potential opportunities for secondary access with average duty cycles equal to $18.14 \%$ and $12.89 \%$ respectively. However, the whole band from 235 to $400 \mathrm{MHz}$ is exclusively reserved for security services and systems of the Spanish Ministry of Defense, which in principle precludes the use of such spectrum bands by CRNs. Other bands below $1 \mathrm{GHz}$ with low or moderate levels of activity but narrower available free bandwidths are those assigned to wireless microphones and RFID (862-870 MHz), CT1 cordless phones (870-871 and 915$916 \mathrm{MHz}$ ), cellular access rural telephony (874-876 and 919$921 \mathrm{MHz}$ ), R-GSM 900 (876-880 and 921-925 MHz), trunking systems (223-235 MHz) and several fixed and mobile services (400-470 MHz). The lower spectrum bands, assigned to FM broadcasting (87.5-108 MHz), maritime and aeronautical radionavigation (108-174 MHz) and audio broadcasting (174$223 \mathrm{MHz}$ ) show intensive usage with average duty cycles between $90 \%$ and $100 \%$.

Above $1 \mathrm{GHz}$ the highest spectrum usage is observed for the bands allocated to the mobile cellular communication systems DCS 1800 (1710-1785 MHz and 1805-1880 MHz) and UMTS (1920-1980 MHz and 2110-2170 MHz). Note that the differences between uplink and downlink usage patterns that were appreciated for E-GSM 900 are also observed for DCS 1800 and UMTS. In the case of DCS 1800 the differences are more accentuated due to the fact that mobile stations in DCS 1800 have a lower transmission power than in GSM 900, which results in a reduced duty cycle in uplink (3.52\%). In the case of UMTS the difference is higher due to the spread spectrum nature of the WCDMA radio technology employed by UMTS. WCDMA signals are modulated over larger bandwidths, which results in very low transmission powers. Such signals might not be detectable by a spectrum analyzer. As a result, very low activity was recorded in the UMTS uplink (2.86\%). Although in the downlink direction DCS 1800 and UMTS show a higher level of activity (59.75\% and $48.38 \%$ respectively), these bands also provide some opportunities for secondary access. In the case of DCS 1800 some portions of the downlink band show a well defined periodic usage pattern 
(higher occupancy between 0-12 and 24-36 hours) that could be exploited by secondary CRNs. In the case of UMTS, several $5 \mathrm{MHz}$ channels appear to be unoccupied. Moreover, the bands reserved for UMTS TDD (1900-1920 and 2010-2025 MHz) and the extension of UMTS $(2500-2690 \mathrm{MHz})$ are not used. The ISM-2450 band (2400-2500 MHz) also appears to be unused in the measurement location, which could be explained by the fact that this frequency band is usually occupied in indoor environments and signals at such frequencies are severely attenuated by walls. The rest of spectrum between 1 and $3 \mathrm{GHz}$ remains mostly unused, with the exception of some signals with very low duty cycle in bands allocated to aeronautical and satellite radiolocation and radionavigation, (960-1350 and 1610-1710 MHz), DECT cordless phones (1880-1900 MHz) and military radars (2700-2900 MHz).

Figure 4 summarizes the band by band average spectrum occupancy statistics. The obtained results demonstrate that some spectrum bands are subjected to intensive usage while some others show moderate utilization, are sparsely used and, in some cases, are not used at all. In general, the average spectrum occupancy observed in frequency and time in this study is significantly low, concretely $22.57 \%$ for the whole frequency range between $75 \mathrm{MHz}$ and $3 \mathrm{GHz}$. However, it is worth noting that the actual spectrum occupancy might be even lower due to the frequency span considered in this study (500 $\mathrm{MHz}$ ). For a given number of frequency points measured per span by a spectrum analyzer, the frequency bin size, i.e. the separation between two consecutive measured frequency points, increases as the measured frequency span increases. If the frequency bin is larger than the bandwidth of the signal being measured, the spectrum occupancy is overestimated as it is shown in Figure 5. As it can be appreciated in the upper graph for DCS 1800 (channel bandwidth of $200 \mathrm{kHz}$ ) a frequency bin of $756 \mathrm{kHz}$ results in a spectrum occupancy overestimation equal to $8.49 \%$ with respect to a frequency bin of $58 \mathrm{kHz}$. The spectrum occupancy is not significantly overestimated as long as the frequency bin size remains acceptably lower than the signal bandwidth. As it can be observed in the lower graph of Figure 5, reducing the frequency bin from $756 \mathrm{kHz}$ to $90 \mathrm{kHz}$ when measuring UMTS signals (channel bandwidth of $5 \mathrm{MHz}$ ) does not change the occupancy value significantly. In order to maintain the measurement period required for the whole frequency range $75-3000 \mathrm{MHz}$ within feasible limits we selected in this study a frequency span equal to $500 \mathrm{MHz}$, which lead to frequency bin sizes larger than the signal bandwidths of several spectrum bands. As a result, the spectrum occupancy statistics obtained in this study should be interpreted as upper bounds of the actual spectrum occupancy. This circumstance highlights the low degree to which spectrum is actually used.

\section{CONCLUSIONS AND FUTURE WORK}

This paper presented spectrum occupancy measurements conducted in the frequency range $75-3000 \mathrm{MHz}$ in an outdoor environment in urban Barcelona, Spain. The obtained results demonstrated the existence of significant amounts of spectrum potentially available for the future deployment of cognitive radio networks, especially between 1 and $3 \mathrm{GHz}$. As a part of our future work we plan to extend our measurement campaign to different environments and scenarios covering the range up to $7 \mathrm{GHz}$ with more sophisticated and accurate measurement setups. These future studies would enable a more realistic understanding of dynamic spectrum utilization and would help us to more accurately identify the most suitable and interesting bands for the future deployment of cognitive radio networks.

\section{ACKNOWLEDGMENTS}

The authors wish to acknowledge the activity of the Network of Excellence in Wireless COMmunications NEWCOM++ of the European Commission (contract n. 216715) that motivated this work. This work has been supported by the Spanish Research Council under COGNOS grant (ref. TEC2007-60985). The support from the Spanish Ministry of Science and Innovation (MICINN) under FPU grant AP2006-848 is hereby acknowledged.

\section{REFERENCES}

[1] I. F. Akyildiz, W.-Y. Lee, M. C. Vuran, and S. Mohanty, "A survey on spectrum management in cognitive radio networks," IEEE Communications Magazine, vol. 46, no. 4, Apr 2008, pp. 40 - 48.

[2] F. H. Sanders, "Broadband spectrum surveys in Denver, CO, San Diego, CA, and Los Angeles, CA: methodology, analysis, and comparative results,” in Proc. 1998 IEEE International Symposium on Electromagnetic Compatibility, Aug 1998, vol. 2, pp. 988-993.

[3] Shared Spectrum Company, "Spectrum occupancy measurements," Shared Spectrum Company reports (Jan 2004 - Aug 2005). Available at: http://www.sharedspectrum.com/measurements/.

[4] A. Petrin, P. G. Steffes, "Analysis and comparison of spectrum measurements performed in urban and rural areas to determine the total amount of spectrum usage,” in Proc. International Symposium on Advanced Radio Technologies (ISART 2005), Mar 2005, pp. 9-12.

[5] P. G. Steffes, A. J. Petrin, "Study of spectrum usage and potential interference to passive remote sensing activities in the $4.5 \mathrm{~cm}$ and $21 \mathrm{~cm}$ bands," in Proc. IEEE International Geoscience and Remote Sensing Symposium (IGARSS 2004), Sep 2004, vol. 3, pp. 1679-1682.

[6] J. Do, D. M. Akos, P. K. Enge, "L and S bands spectrum survey in the San Francisco bay area,” in Proc. Position Location and Navigation Symposium (PLANS 2004), Apr 2004, pp. 566-572.

[7] M. Biggs, A. Henley, T. Clarkson, “Occupancy analysis of the $2.4 \mathrm{GHz}$ ISM band,” IEE Proc. on Comms, Oct 2004, vol. 151, n 5, pp. 481-488.

[8] S. W. Ellingson, "Spectral occupancy at VHF: implications for frequency-agile cognitive radios,” in Proc. IEEE 62nd Vehicular Technology Conference (VTC 2005-Fall), Sep 2008, vol. 2, pp. 1379-1382.

[9] S. D. Jones, E. Jung, X. Liu, N. Merheb, I.-J. Wang, "Characterization of spectrum activities in the U.S. public safety band for opportunistic spectrum access," in Proc. 2nd IEEE International Symposium on New Frontiers in Dynamic Spectrum Access Networks (DySPAN 2007), Apr 2007, pp. 137-146.

[10] R. I. C. Chiang, G. B. Rowe, K. W. Sowerby, "A quantitative analysis of spectral occupancy measurements for cognitive radio,” in Proc. IEEE 65th Vehicular Technology Conference (VTC 2007-Spring), Apr 2007, pp. 3016-3020.

[11] M. H. Islam et al., "Spectrum Survey in Singapore: Occupancy Measurements and Analyses,” in Proc. 3rd International Conference on Cognitive Radio Oriented Wireless Networks and Communications (CrownCom 2008), May 2008, pp. 1-7.

[12] M. Wellens, J. Wu, P. Mähönen, "Evaluation of spectrum occupancy in indoor and outdoor scenario in the context of cognitive radio," in Proc. Second International Conference on Cognitive Radio Oriented Wireless Networks and Communications (CrowCom 2007), Aug 2007, p. 8.

[13] State Agency for Telecommunications and Information Society, "Cuadro Nacional de Atribución de Frecuencias (CNAF)," Technical Report (in Spanish), 15 Nov 2007. Available online at: http://www.mityc.es/Telecomunicaciones/Secciones/Espectro/cnaf/.

[14] A. Sahai, N. Hoven, R. Tandra, "Some fundamental limits on cognitive radio," in Proc. Forty-second Allerton Conference on Communications, Control, and Computing (Allerton Conference 2004), Sep 2004, p. 10. 

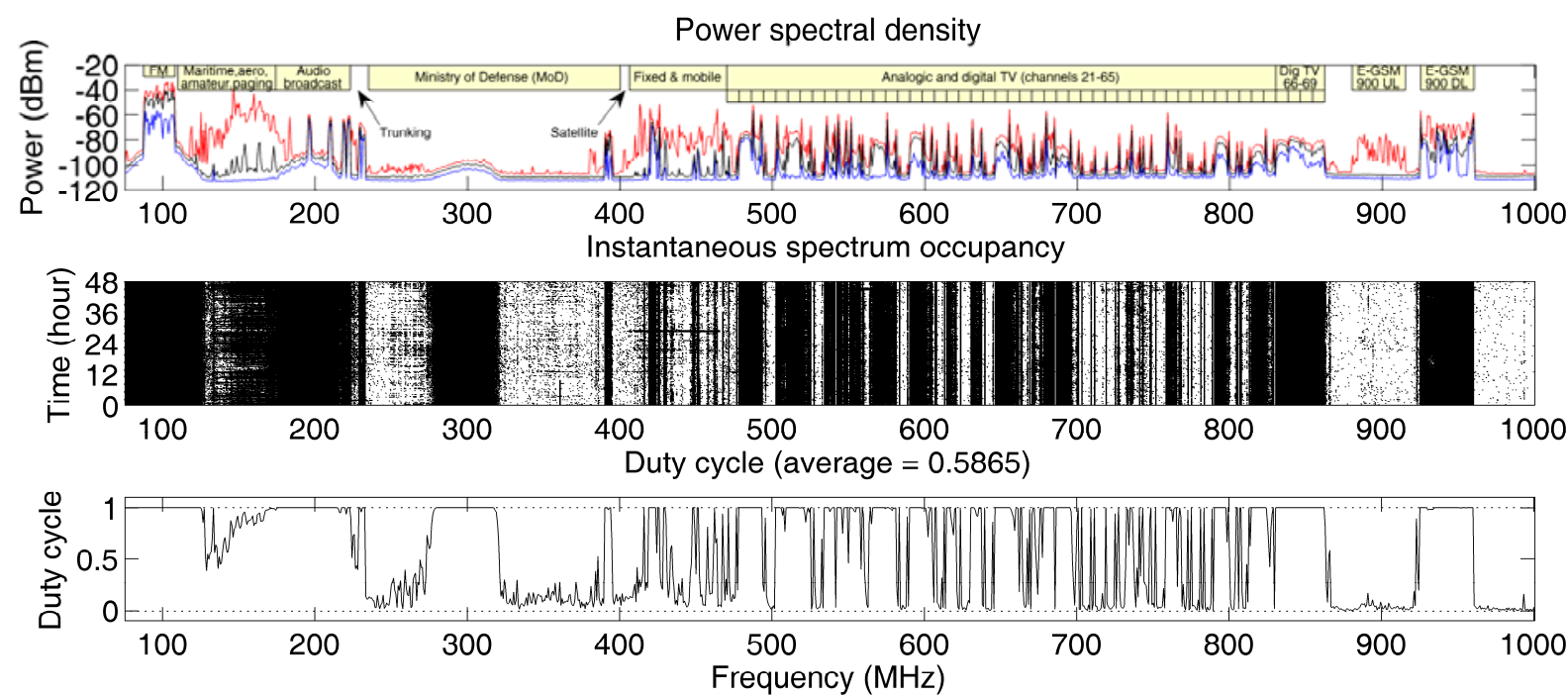

Figure 2. Occupancy results between 75 and $1000 \mathrm{MHz}$.
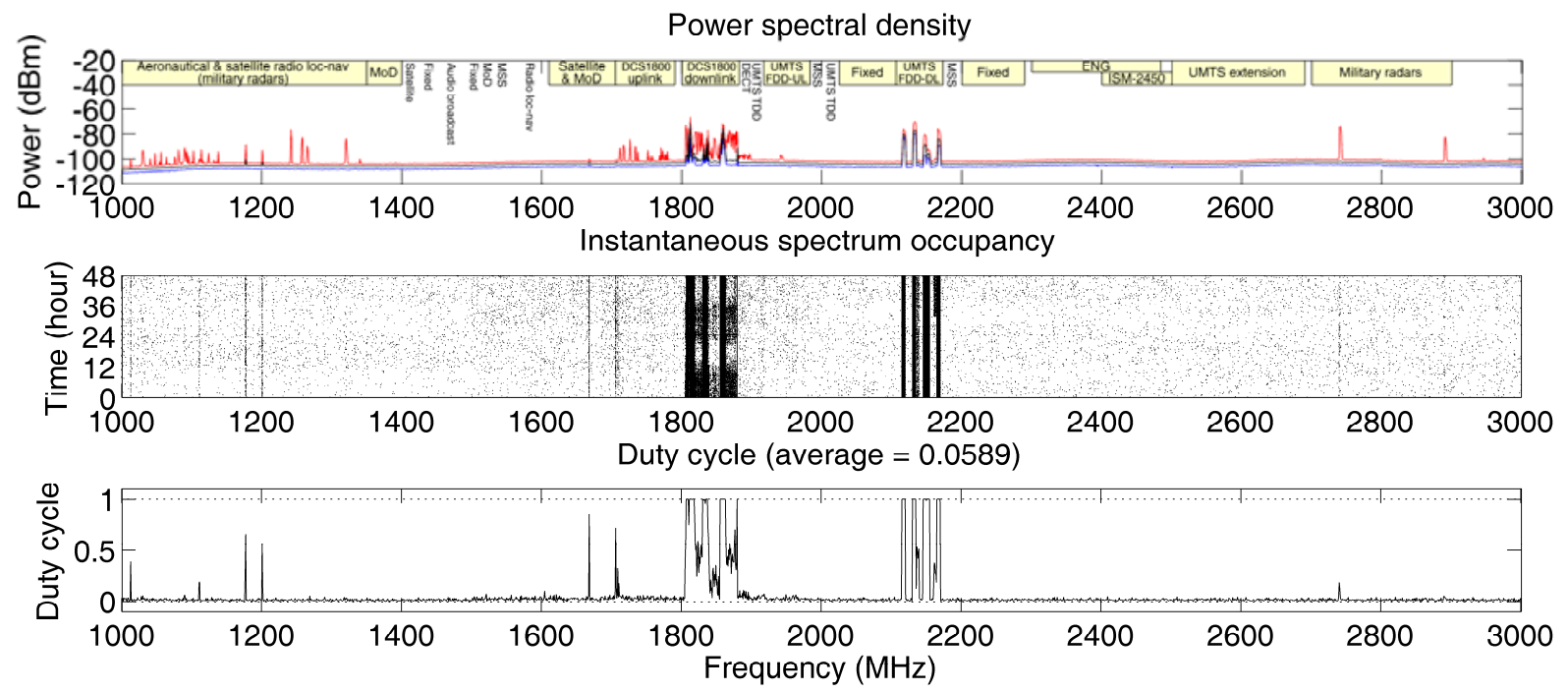

Figure 3. Occupancy results between 1000 and $3000 \mathrm{MHz}$.

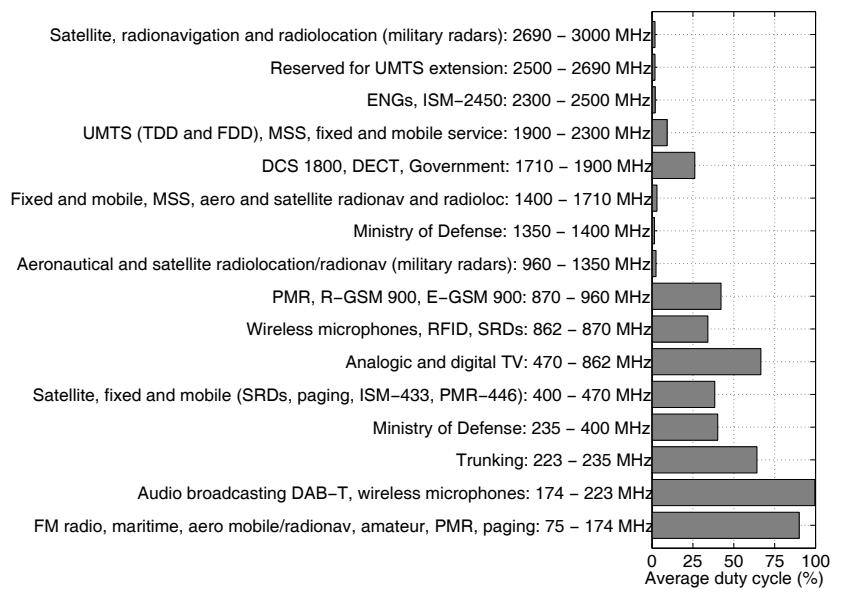

Figure 4. Band by band average duty cycle statistics.
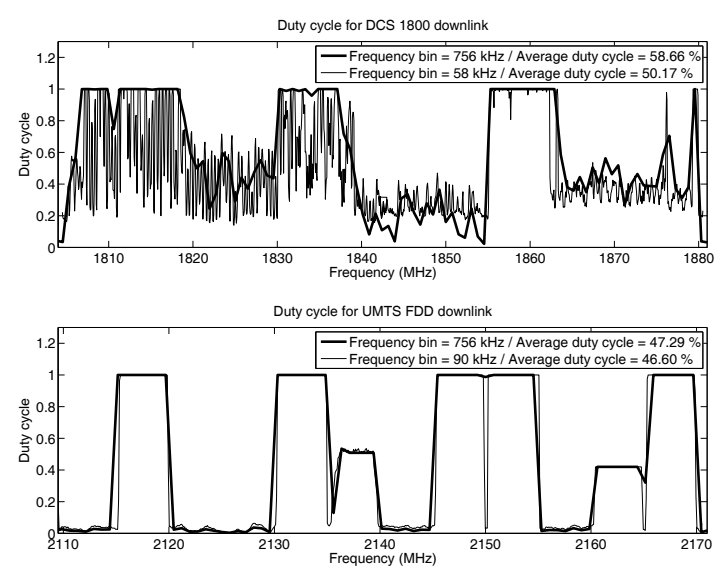

Figure 5. Effect of the frequency bin on the estimated spectrum occupancy. 